CLINICAL STUDY

\title{
Changes in calf muscle elasticity in hypogonadal males before and after testosterone substitution as monitored by magnetic resonance elastography
}

\author{
Katja Brauck, Craig J Galbán, Stefan Maderwald, Burkhard L Herrmann ${ }^{1}$ and Mark E Ladd \\ Department of Diagnostic and Interventional Radiology and Neuroradiology, University Hospital Essen, Hufelandstrasse 55, 45122 Essen, Germany and \\ ${ }^{1}$ Department of Internal Medicine, Endocrinology, University Hospital Essen, Essen, Germany \\ (Correspondence should be addressed to K Brauck; Email: katja.brauck@uni-duisburg-essen.de)
}

\begin{abstract}
Purpose: We sought to determine whether differences in muscle elasticity between healthy age-related controls and patients with hypogonadism could be measured by a new, non-invasive method termed magnetic resonance elastography (MRE).

Materials and methods: Twelve hypogonadal (21-68 years) and twenty-seven healthy age-related men (20-76 years) were examined. In the hypogonadism group, serum testosterone levels were compared and MRE was conducted prior to and after 6 months of therapy in 6 of the 12 patients. MRE was performed by mechanically exciting the soleus muscle that was used because of its uniformly distributed muscle fibers, size, and accessibility, with a custom designed piezoelectric-actuator using a modified phase-contrast sequence. For mechanical excitation the actuator lever was placed on the anterior surface of the calf. The subjects had to maintain a force of $0-20 \%$ of their maximum voluntary contraction against a home-built footplate that was mounted on the MR table. All images were phase unwrapped and reconstructed into shear modulus elastograms using the local frequency estimation technique.

Results: Testosterone levels were significantly higher after 6 months of treatment. A statistical difference in the shear modulus was observed prior to and after 6 months of testosterone therapy and was nearly the same as in the healthy age-related control group.

Conclusion: MRE seems to be a promising technique for the evaluation of therapeutic effects in patients with hypogonadism and possibly in other diseases with muscular effects.
\end{abstract}

European Journal of Endocrinology 156 673-678

\section{Introduction}

Male hypogonadism is characterized by androgen deficiency and infertility. Hypogonadism can be caused by disorders at the hypothalamic or pituitary level (hypogonadotropic form) or by testicular dysfunction (hypergonadotropic form). It can be a congenital failure or acquired during life. The reduction of testosterone concentrations presents manifold effects in these patients: slight anemia, changes in coagulation parameters, regression of sexual function, decreased bone density, muscle atrophy, and increased body fat concentration are some of the notable changes $(1,2)$.

Lack of testosterone, as it occurs in hypogonadal men, leads to loss of muscle strength and size and reduction in laboratory parameters that estimate muscular metabolism (3). The effects of testosterone substitution on the skeletal musculature have been well studied in recent years. Application of a controlled dosage of testosterone in hypogonadal men over a period of approximately 6 months improves muscle strength, increases muscle size, and restores anabolic muscular metabolism to nearly normal levels $(4,5)$.

Effects of testosterone substitution on the skeletal musculature were measured in these studies by laboratory tests, which estimate the state of the muscular metabolism, and by various imaging techniques, e.g. dual energy X-ray absorptiometry (1) or magnetic resonance imaging (MRI; (4)).

In recent years, the introduction of innovative MRI techniques has opened up new possibilities for improving the use of MRI for monitoring patient response to testosterone therapy. Magnetic resonance elastography (MRE) is a novel approach for measuring muscle stiffness during contraction $(6,7)$. For image contrast, this technique utilizes the elastic properties of tissue, which vary between different tissues or between different affections of one tissue. In its most straightforward application under several simplifying assumptions for the reconstruction algorithm (isotropy, homogeneity, and lack of viscous damping), MRE is able to determine the shear modulus $(\mathrm{kPa})$ of the examined tissue. 
Using a phase-contrast (PC)-based MRI imaging technique, MRE visualizes and quantitatively measures propagating acoustic strain waves in tissues generated by harmonic mechanical excitation (vibration). The strain (deformation per unit length) is the deformation of materials caused by stress (force per unit area). For example, a harmonic mechanical excitation caused by an external vibratory device induces an oscillating stress, which in turn induces a wave of displacement through the medium. Since the frequencies applied are in the acoustic range $(50-500 \mathrm{~Hz})$, they are generally termed acoustic strain waves. The acquired data allow the calculation of local quantitative values of shear modulus and the generation of images that depict tissue elasticity or stiffness (8).

Previous studies of muscle tissue elasticity using MRE have shown that muscle stiffness increases with increasing load $(9,10)$. Muscle stiffness has also been shown to vary between muscle groups, gender (7), and degree of muscle load (11). A preliminary study comparing healthy subjects to a small group of patients with neuromuscular dysfunction in the lower extremities with varying etiology (6) demonstrated differences in muscle stiffness between the two groups.

In this study, the effect of testosterone substitution was evaluated by MRE. We examined the calf muscles of patients with hypogonadism prior to testosterone substitution and following 6 months of treatment. These patients were compared with age-related healthy volunteers. To eliminate potential effects on muscle stiffness due to differences in age, we examined an agerelated group of healthy volunteers over the same time period. We hypothesized that muscle stiffness of hypogonadal males would significantly increase after 6 months of therapy and obtain levels comparable with the range of healthy subjects, whereas the control group would not show any differences in muscle stiffness to their prior results.

\section{Materials and methods}

\section{Subjects}

The study was conducted in accordance with the regulations of the local ethics committee. Written informed consent was obtained prior to the examination from all participants including patients and healthy volunteers. The study participants were 12 hypogonadal men, 21-68 years of age (mean 38 years \pm 15 (s.D.)) and otherwise in good health, with testosterone levels less than $10.4 \mathrm{nmol} / \mathrm{l}$ (normal range $12-30 \mathrm{nmol} / \mathrm{l}$ ). Five patients had a history of primary hypogonadism and seven patients of secondary hypogonadism. None of the subjects had been previously treated with testosterone. The patients were treated with $50 \mathrm{mg}$ testosterone gel daily. A 6-month follow-up MRE examination was performed only on six patients. The remaining patients were not available due to cessation of treatment at our institution. In addition, 27 healthy age-related volunteers with no signs of hypogonadism were included in the study (range 20-76 years of age, mean 43 years \pm 3 (s.D.)). There was no statistical difference between the ages of the two groups (unpaired two sided $t$-test).

Serum testosterone levels were monitored in the hypogonadal group through standard laboratory blood test both immediately preceding treatment commencement and after 6 months of testosterone substitution.

\section{MRI experiment}

All MRI experiments were performed on a $1.5 \mathrm{~T}$, wholebody system (Magnetom Sonata, Siemens AG, Erlangen, Germany) equipped with a gradient system capable of $40 \mathrm{mT} / \mathrm{m}$ maximum amplitude and a slew rate of $200 \mathrm{mT} / \mathrm{m}$ per ms. The subjects lay supine on the MR table with their feet securely fastened to a home-built footplate. The footplate, which was used to measure applied force through a strain gauge, was fixed at a $90^{\circ}$ angle. A linearly polarized six-channel body coil (Siemens) was used for signal reception. For the mechanical excitation of the muscle tissue, a Plexiglas actuator lever was placed on the anterior surface of the calf approximately $5 \mathrm{~cm}$ below the knee (Fig. 1). Prior to scanning, the patients were instructed to keep their knees fully extended and to apply a force only by performing a plantar flexor motion with both feet. This guaranteed that only muscles in the calf would be used during the experiment. While the subjects were being

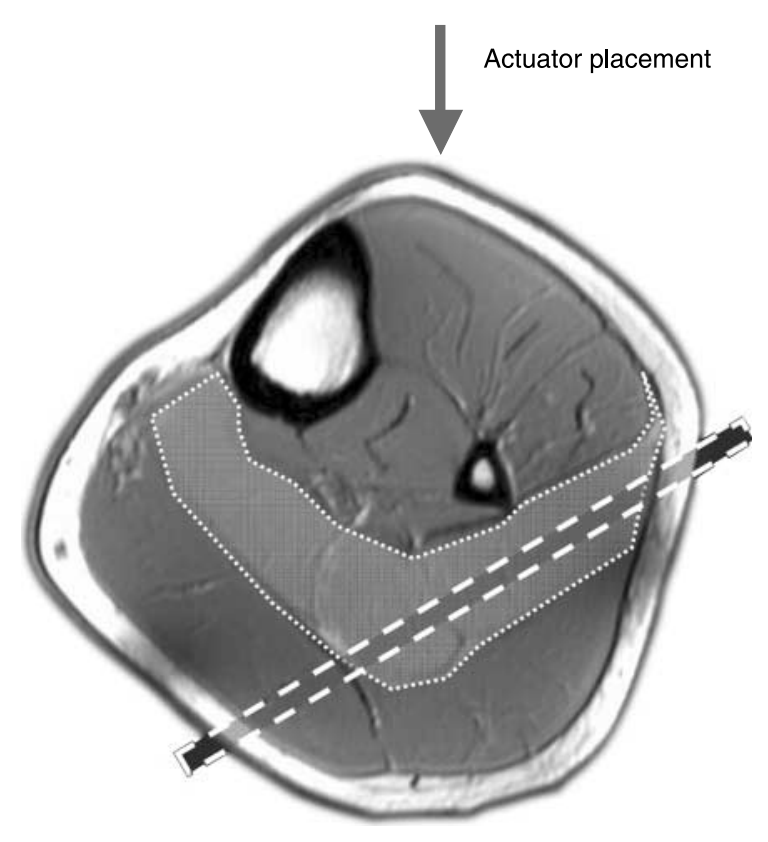

Figure 1 Anatomical image of the soleus (irregular region of interest) showing the slice acquired oblique coronal along the orientation of the muscle (rectangle) and point of mechanical excitation (arrow). 
informed of the experimental procedure, the force (measured in volts) exerted by the subject in a relaxed state was calibrated to zero. The subjects were then informed to exert their maximum force for $2 \mathrm{~s}$. No motivational speech was used during this measurement. This procedure was repeated three times, and the maximum voltage of all three attempts was designated the voltage at maximum voluntary contraction (MVC). The maximum voltage was used for calculating the percentage of the MVC $(\mathrm{pMVC}=100 \times$ voltage $/$ maximum voltage). This value was displayed on a personal computer and on an LED device (Fig. 2) that was placed within the MRI room, which was visible to the subjects throughout the experiment.

All subjects were advised to relax during localization and volumetric scans. The localization scans were initially performed to identify and locate the region of interest (ROI) of the soleus muscle for the volumetric scan. The volumetric scan was used to display the whole muscle volume for planning the following five MRE scans. For MRE, a modified PC gradient echo sequence equipped with trapezoidal motion-sensitizing gradients was employed (repetition time (TR) $120 \mathrm{~ms}$, echo time (TE) $33.3 \mathrm{~ms}$, flip angle $15^{\circ}$, matrix $128 \times 80$, field of view (FOV) $270 \times 180 \mathrm{~mm}^{2}$, bandwidth $260 \mathrm{~Hz} /$ pixel, slice thickness $5 \mathrm{~mm}$, and special parameters of the
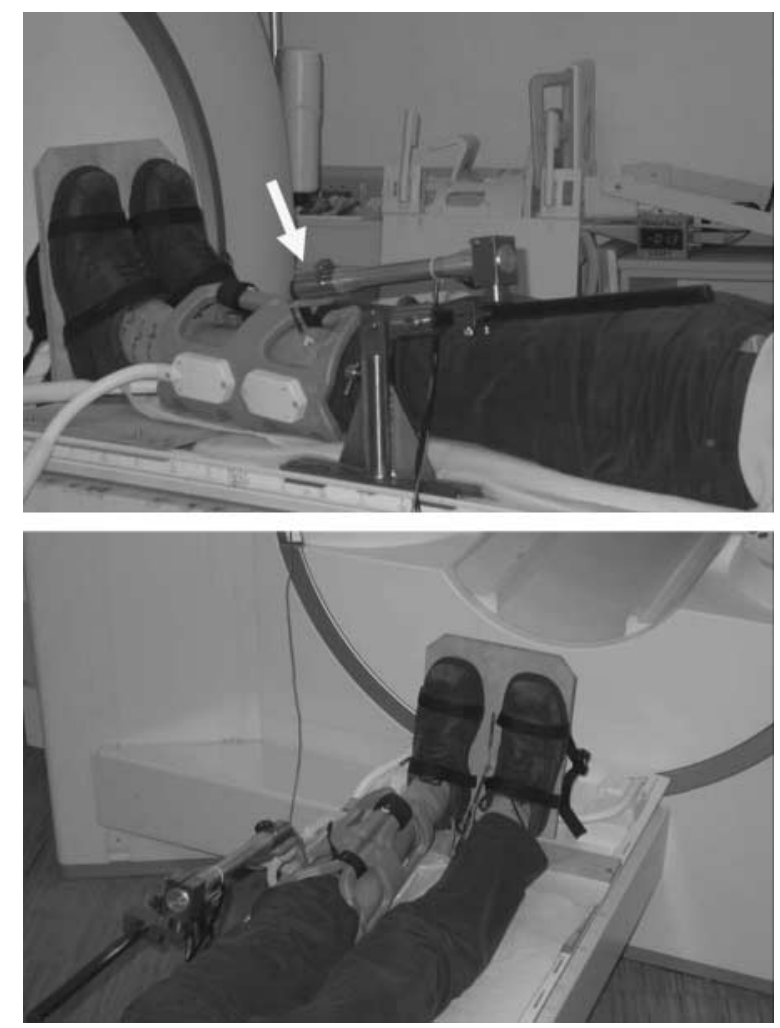

Figure 2 Setup showing the six-element body array coil for imaging the soleus muscle, the piezoelectric mechanical oscillator (arrow), and the footplate for recording muscle force. modified PC sequence: three trapezoidal motion-sensitizing gradient cycles with $25 \mathrm{mT} / \mathrm{m}$ amplitude).

During the individual MRE scans, the subjects were required to maintain a force on the footplate of $0,5,10$, 15 , and $20 \%$ of their MVC for $\sim 80 \mathrm{~s}$. All force measurements were recorded on a personal computer and were later analyzed to determine if the target force was maintained during the MRE scans.

\section{MR elastography}

For the MRE experiment, the mechanical waves were induced into the calf by mechanically exciting the tissue with a custom-designed piezoelectric vibrator (12). The mechanical oscillation was generated in phase with the motion-sensitizing gradients. Stability of the phase relationship was maintained by a trigger, which was sent every TR from the MR scanner to a digital signal processing (DSP) card in a PC. Discrete phase offsets were realized by adding a fixed offset to the trigger pulse. The DSP card synchronized the control voltage for driving the actuator piezoelectric ceramic to the trigger signal in such a way that continuous sinusoidal oscillation was possible throughout the experiment.

Imaging of the soleus muscle was performed with the Plexiglas actuator lever located as described above with an excitation frequency of $100 \mathrm{~Hz}$, and the vibration amplitude of the lever was in the range of 600-700 $\mu \mathrm{m}$. The $100 \mathrm{~Hz}$ excitation frequency ensured adequate wave penetration into the soleus with a sufficient number of wave cycles in the ROI for reconstruction. The soleus muscle was considered because of its uniformly distributed muscle fibers, size, and accessibility. The vibration was orientated in the anterior/ posterior direction. All elastography images were oriented oblique coronally along the orientation of the muscle with the motion encoding parallel to the mechanical oscillation. For a complete dataset, a total of eight equidistant phase offsets $\left(0^{\circ}, 45^{\circ}, 90^{\circ}, 135^{\circ}\right.$, $180^{\circ}, 225^{\circ}, 270^{\circ}, 315^{\circ}$ ) were acquired. Figure 2 shows the setup for imaging the soleus muscle.

\section{Data analysis}

All phase images were phase unwrapped and reconstructed into shear modulus elastograms assuming that the muscle tissue was homogeneous. For the reconstructions, the local frequency estimation (13) technique was used to determine shear wavelength and from this to estimate the shear modulus of the muscle $(7,14)$. The underlying principle is based on the fact that the wave propagation velocity is higher in stiffer materials. A longer wavelength (lower local frequency) therefore indicates a stiffer material (Fig. 3). A muscle tissue density of $1100 \mathrm{~kg} / \mathrm{m}^{3}$ was assumed. The resultant shear modulus elastograms were then averaged over all eight phase offsets to a final elastogram. Regions of interest were manually chosen from the final 


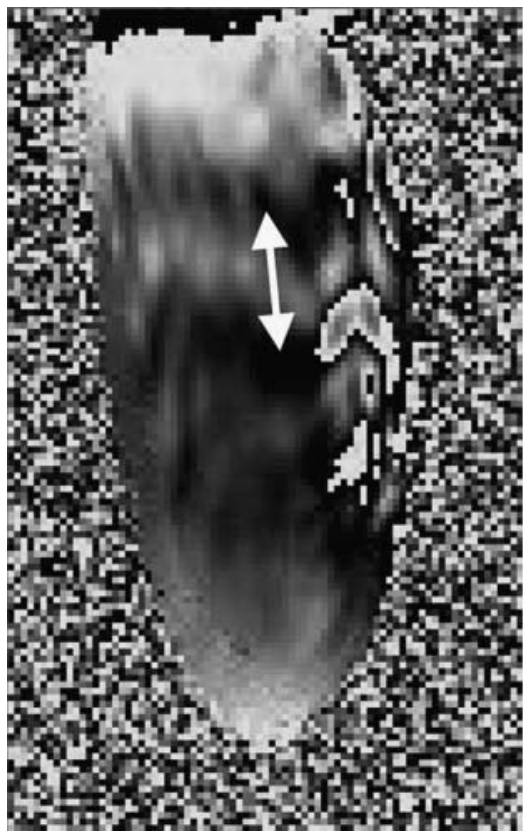

$0 \% \mathrm{MVC}$

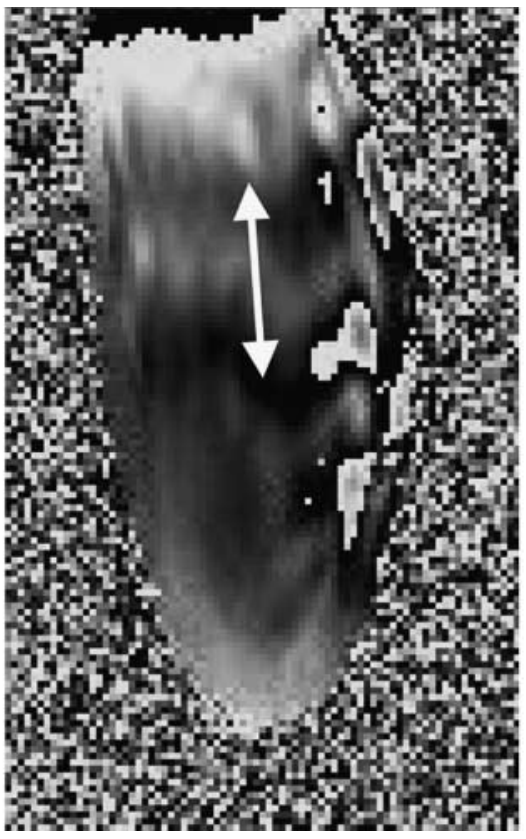

$10 \%$ MVC

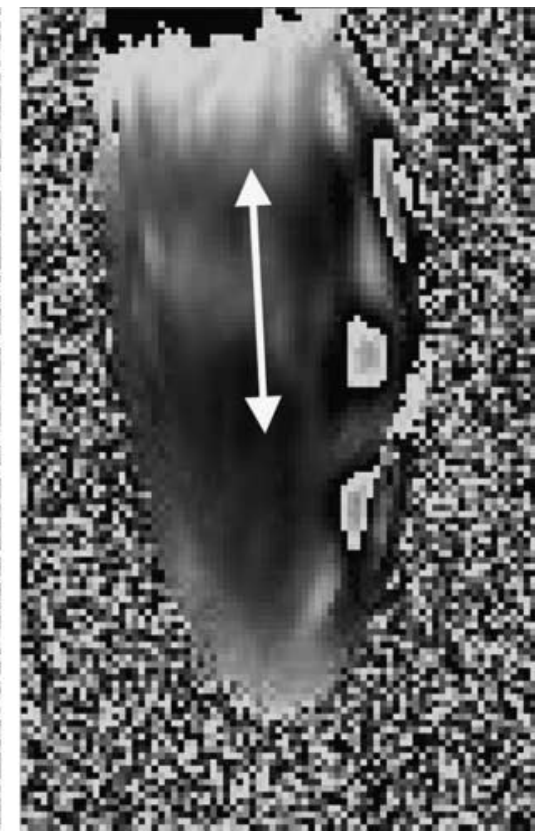

$20 \%$ MVC

Figure 3 Shear wave images at 0,10 , and $20 \%$ of MVC (maximum voluntary contraction; pMVC $=100 \times$ voltage/maximum voltage). The increasing wavelength indicates increasing shear modulus (stiffness).

elastogram to calculate the mean shear modulus of the soleus muscle. The soleus muscle displayed the required uniform wave excitation and anatomy at this frequency in contrast to other muscle groups. The wave patterns in the phase images were taken into account to exclude areas at muscle boundaries where the complexity of the wave patterns is extreme due to reflections and refraction, which leads to distortions of the local wavelength. When possible, the ROI was chosen to include several wave cycles in the phase images.

The shear modulus data in the soleus muscle were plotted as a function of \%MVC. A two-sided unpaired $t$-test was used to determine statistical differences in the shear moduli for a specific MVC between the pre-treatment hypogonadism and post-treatment hypogonadism groups. A similar two-sided unpaired $t$-test was performed for a specific MVC between the pretreatment hypogonadism and healthy volunteer groups, and between the post-treatment hypogonadism and healthy volunteer groups. Hormone levels were analyzed using a two-sided unpaired $t$-test. Data were given as mean value \pm standard error of the mean (s.E.M.). Statistical significance was assessed at $P<0.05$.

\section{Results}

\section{Hormone levels}

Serum testosterone levels of the hypogonadal men increased significantly from a pre-treatment level of
$6.12 \pm 0.89$ to $22.80 \pm 10.67 \mathrm{nmol} / \mathrm{l}$ (mean \pm s.D) Serum testosterone levels are shown in Fig. 4.

\section{MR elastography}

MRE was possible in all participants with no serious side effects. The shear modulus data of the soleus muscle for $0,5,10,15$, and $20 \%$ in the hypogonadism group pretreatment were lower than in the age-related healthy control group. For 5 and $20 \%$ of MVC, a statistical significance was observed. After 6 months of testosterone substitution treatment, the shear modulus values

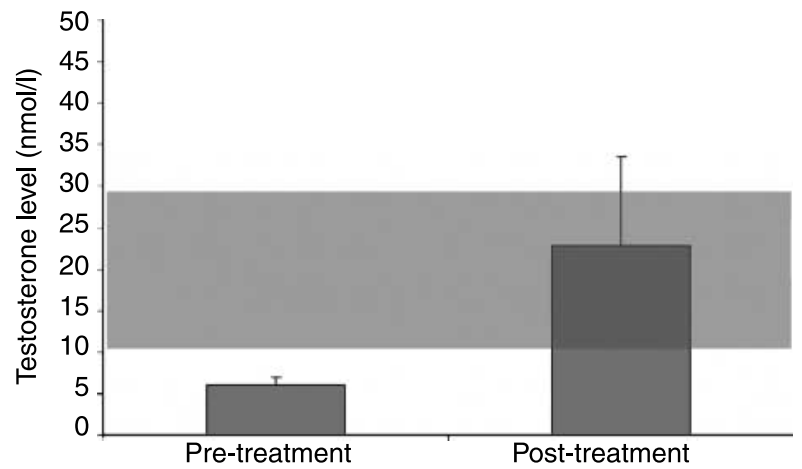

Figure 4 Serum testosterone levels (nmol/l) in the hypogonadal group prior to testosterone treatment $(n=12)$ and after 6 months of treatment $(n=6)$. Bar shows normal range of testosterone levels $(12-30 \mathrm{nmol} / \mathrm{l})$. Data presented as mean \pm S.E.M. 


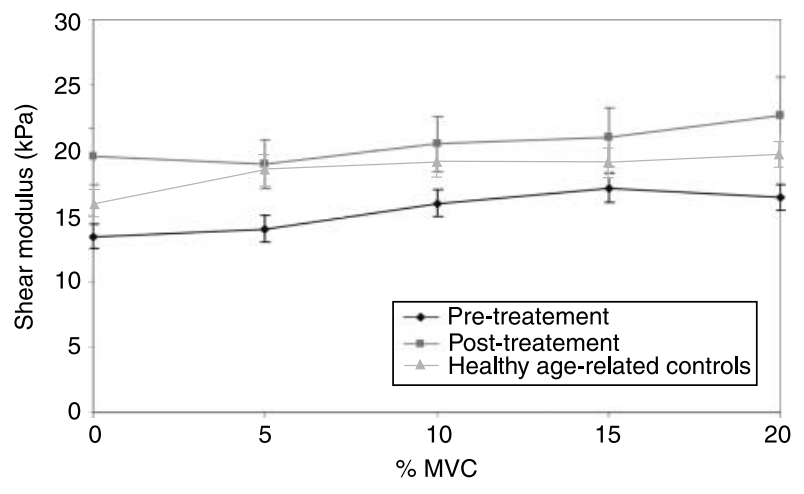

Figure 5 Shear modulus $(\mathrm{kPa})$ at various percentages of MVC in patients pre-treatment and 6 months post-testosterone treatment and in healthy age-related volunteers. Data are presented as mean \pm S.E.M.

increased and a statistical significance was observed for $0,5,10$, and $20 \%$ in comparison with shear modulus values prior to testosterone therapy. The shear modulus data were also slightly higher in the treated group than in the age-related healthy volunteer group (but not statistically significant). The shear modulus data are shown in Fig. 5 and Table 1.

\section{Discussion}

In recent years, the anabolic effects of testosterone on muscle mass and body composition have been determined by various investigators. They observed that testosterone substitution leads to an increase in muscle mass and muscle strength due to hypertrophy of muscle fibers, while at the same time leading to a decrease in body fat content $(2,4,15,16)$. In some of these studies, MRI was used to determine morphologic changes of the musculature. However, additional examination methods were required to obtain a complete picture of the expected testosterone effects.

The development of MRE offers the possibility to gain more information from the MR examination because it reveals changes in muscle elasticity. Muscle elasticity, which characterizes the deformation of muscle tissue under stress (e.g. external forces), depends strongly on muscle properties, e.g. muscle fiber density, muscle strength, muscle endurance, and intramuscular fat (9). Changes in these components lead to variances in muscle stiffness and consequently to changes in the shear modulus of the examined muscle. Muscle elasticity changes secondary to disease processes have already been detected by MRE. Basford et al. (6) showed in one of the first in vivo experiments a statistically significant difference in muscle stiffness between healthy volunteers and patients with neuromuscular dysfunction.

Uffmann et al. (7) observed no significant variations in muscle elasticity measured in the same muscle group at different time points in a group of healthy volunteers, but up to now MRE has not been used to assess therapeutic effects over a period of time in patients. In our study, hypogonadal patients were observed over a period of 6 months. The results show very clearly the effect of testosterone substitution on muscle stiffness. Prior to treatment, the shear modulus data were significantly lower than in the healthy control group. After 6 months of treatment, the data showed complete normalization, even showing slightly higher muscle stiffness than in the healthy control group.

There remains uncertainty about how to properly compare MRE results obtained by different investigator groups. There is currently considerable variation in shear modulus data between different groups, making absolute quantitative comparison between these data difficult (7). The variations may stem from differences in experimental setup or in the exact method used to reconstruct the data. The placement of the actuator on different muscles groups, e.g. biceps muscle or calf muscles, inherently delivers a range of results because of differing anisotropy in different soft tissues (17). Hamhaber et al. (18) showed these elasticity properties of soft tissue in a model using agar phantoms with different agar concentrations. The results show that the elasticity range of the gels covers the elasticity range of typical soft tissues. For the data presented in this study, we used the same setup throughout. The low standard errors obtained seem to confirm the consistency of the experimental setup; however, it is still unclear how much of the variation resulted from remaining inconsistencies in the data acquisition. Also, although the soleus muscle was chosen for its uniform wave propagation, this wave appearance alone does not guarantee that boundary effects are not present. Thus, some error may have been introduced due to the simplifying assumptions of the MRE reconstruction algorithm used.

Table 1 Shear modulus $(\mathrm{kPa})$ in the healthy control group as well as prior to and after 6 months of testosterone treatment in the hypogonadal group. Data are presented as mean \pm s.E.M.

\begin{tabular}{lccccc}
\hline & 0\% MVC & 5\% MVC & 10\% MVC & 15\% MVC & 20\% MVC \\
\hline Pre-treatment & $13.42 \pm 0.92^{\mathrm{a}}$ & $14.02 \pm 1.05^{\mathrm{a}, \mathrm{b}}$ & $15.96 \pm 1.03^{\mathrm{a}}$ & $17.11 \pm 1.13$ & $16.44 \pm 0.99^{\mathrm{a}, \mathrm{b}}$ \\
Post-treatment & $19.54 \pm 2.19$ & $18.93 \pm 1.85$ & $20.49 \pm 2.09$ & $21.00 \pm 2.28$ & $22.63 \pm 3.01$ \\
Healthy control & $15.96 \pm 1.00$ & $18.52 \pm 1.26$ & $19.18 \pm 1.21$ & $19.13 \pm 1.11$ & $19.75 \pm 0.99$ \\
\hline
\end{tabular}

${ }^{\text {aS }}$ tatistical significance pre- versus post-treatment.

${ }^{b}$ Statistical significance pre-treatment versus age-related healthy controls. 
When looking at the data in Fig. 5, the only data point that deviates from the general trend in the three groups is the shear modulus at $0 \%$ MVC in the posttreatment group. The data for $0 \%$ MVC should be viewed with care in all three groups, since it is very difficult to define the $0 \%$ MVC state, as the muscle elasticity varies with isometric exertion. Although the subjects were instructed prior to scanning to keep their knees fully extended and to apply a force only by performing a plantar flexor motion with both feet, compliance was not monitored and residual muscle stiffening due to dorsiflexion cannot be strictly ruled out. Although every attempt was made to ensure that the subjects adjusted the pressure on the footplate to obtain zero force and that the calf muscles were in a fully relaxed state, the calf muscles may have been more or less taut in this position. The muscle state at higher $\% \mathrm{MVC}$ is much better defined.

MRE seems to be a promising technique for noninvasive evaluation of therapy effects in patients with hypogonadism and possibly in other diseases with muscular effects. Using a consistent setup allows monitoring over several months with consistent results. To our knowledge, this is the first application of this technique to examine hypogonadism, and larger studies will have to be done to confirm our initial experiences and determine the efficacy of MRE in therapy monitoring.

\section{Acknowledgements}

This work was supported by a grant from the Ministry for Innovation, Science, Research and Technology of the state of North Rhine-Westphalia, Germany.

\section{References}

1 Brodsky IG, Balagopal P \& Nair KS. Effects of testosterone replacement on muscle mass and muscle protein synthesis in hypogonadal men - a clinical research center study. Journal of Clinical Endocrinology and Metabolism 199681 3469-3475.

2 Katznelson L, Finkelstein JS, Schoenfeld DA, Rosenthal DI, Anderson EJ \& Klibanski A. Increase in bone density and lean body mass during testosterone administration in men with acquired hypogonadism. Journal of Clinical Endocrinology and Metabolism $1996 \mathbf{8 1} 4358-4365$.

3 Mauras N, Hayes V, Welch S, Rini A, Helgeson K, Dokler M, Veldhuis JD \& Urban RJ. Testosterone deficiency in young men: marked alterations in whole body protein kinetics, strength, and adiposity. Journal of Clinical Endocrinology and Metabolism $1998 \mathbf{8 3}$ 1886-1892.

4 Bhasin S, Storer TW, Berman N, Yarasheski KE, Clevenger B, Phillips J, Lee WP, Bunnell TJ \& Casaburi R. Testosterone replacement increases fat-free mass and muscle size in hypogonadal men. Journal of Clinical Endocrinology and Metabolism $1997 \mathbf{8 2}$ 407-413.

5 Bhasin S, Woodhouse L \& Storer TW. Proof of the effect of testosterone on skeletal muscle. Journal of Endocrinology $20011 \mathbf{1 7 0}$ 27-38.

6 Basford JR, Jenkyn TR, An KN, Ehman RL, Heers G \& Kaufman KR. Evaluation of healthy and diseased muscle with magnetic resonance elastography. Archives of Physical Medicine and Rehabilitation 200283 1530-1536.

7 Uffmann K, Maderwald S, Ajaj W, Galban CG, Mateiescu S, Quick HH \& Ladd ME. In vivo elasticity measurements of extremity skeletal muscle with MR elastography. NMR in Biomedicine 2004 17 181-190.

8 Manduca A, Oliphant TE, Dresner MA, Mahowald JL, Kruse SA, Amromin E, Felmlee JP, Greenleaf JF \& Ehman RL. Magnetic resonance elastography: non-invasive mapping of tissue elasticity. Medical Image Analysis 20015 237-254.

9 Dresner MA, Rose GH, Rossman PJ, Muthupillai R, Manduca A \& Ehman RL. Magnetic resonance elastography of skeletal muscle. Journal of Magnetic Resonance Imaging 2001 13 269-276.

10 Bensamoun SF, Ringleb SI, Littrell L, Chen Q, Brennan M, Ehman RL \& An KN. Determination of thigh muscle stiffness using magnetic resonance elastography. Journal of Magnetic Resonance Imaging 200623 242-247.

11 Bensamoun S, Ringleb S, Chen Q, Ehman R \& An KN. Mechanical properties of relaxed and contracted thigh muscles using magnetic resonance elastography. Computer Methods in Biomechanics and Biomedical Engineering 20051 31-32.

12 Uffman K, Abicht C, Grote W, Quick HH \& Ladd ME. Design of a MR-compatible piecoelectric acutator for MR elastography. Concepts in Magnetic Resonance Part B: Magnetic Resonance Engineering 200215 239-254.

13 Manduca A, Muthupillai R, Rossman PJ, Greenleaf JF \& Ehman RL. Image processing for magnetic resonance elastography. Society of Photo-Optical Instrumentation Engineers 19962710 616-623.

14 Galban CG, Maderwald S, Eggebrecht H, Grote W, de Greiff A, Uffmann K \& Ladd ME. Measuring skeletal muscle elasticity in patients with hypogonadism by MR elastography. Proceedings of the 13th Annual Meeting of ISMRM, 2005.

15 Zitzmann M \& Nieschlag E. Effects of androgen replacement on metabolism and physical performances in male hypogonadism. Journal of Endocrinological Investigation 200326 886-892.

16 Sinha-Hikim I, Artaza J, Woodhouse L, Gonzalez-Cadavid N, Singh AB, Lee MI, Storer TW, Casaburi R, Shen R \& Bhasin S. Testosterone-induced increase in muscle size in healthy young men is associated with muscle fiber hypertrophy. American Journal of Physiology, Endocrinology and Metabolism $20022 \mathbf{2 8 3}$ E154-E164.

17 Gennisson JL, Catheline S, Chaffai S \& Fink M. Transient elastography in anisotropic medium: application to the measurement of slow and fast shear wave speeds in muscles. Journal of the Acoustical Society of America 2003114 536-541.

18 Hamhaber U, Grieshaber FA, Nagel JH \& Klose U. Comparison of quantitative shear wave MR-elastography with mechanical compression tests. Magnetic Resonance in Medicine 200349 $71-77$.

Received 21 November 2006

Accepted 28 March 2007 